\title{
Optimal activation methods for maximizing the concentrations of platelet-derived growth factor-BB and transforming growth factor- $\beta 1$ in equine platelet-rich plasma
}

\author{
Kentaro FUKUDA ${ }^{1}$, Taisuke KURODA ${ }^{1}$, Norihisa TAMURA ${ }^{1}$, Hiroshi MITA ${ }^{1 \text { ) }}$ and \\ Yoshinori KASASHIMA ${ }^{2) *}$ \\ ${ }^{1)}$ Clinical Veterinary Medicine Division, Equine Research Institute, Japan Racing Association, 1400-4 Shiba, \\ Shimotsuke-shi, Tochigi 329-0412, Japan \\ ${ }^{2)}$ The Equine Research Institute, Japan Racing Association, 1400-4 Shiba, Shimotsuke-shi, Tochigi 329-0412, \\ Japan
}

J. Vet. Med. Sci.

82(10): 1472-1479, 2020

doi: 10.1292/jvms.20-0167

Received: 23 March 2020

Accepted: 6 August 2020

Advanced Epub:

19 August 2020

\begin{abstract}
Platelet-rich plasma (PRP) therapy has been widely applied in various medical fields including humans and horses. This study aimed to establish an optimal activation method to stably and reproducibly maximize the concentrations of platelet-derived growth factor-BB (PDGF-BB) and transforming growth factor- $\beta 1$ (TGF- $\beta 1$ ) contained in equine PRP. Autologous PRP was prepared from 11 Thoroughbreds. For the activation test, PRP was activated by either a single freeze-thaw cycle ( $F r)$ or adding calcium and autologous serum containing thrombin (Ca). PDGF$\mathrm{BB}$ and TGF- $\beta 1$ concentrations in $\mathrm{Fr}, \mathrm{Ca}$, nonactivated (No), and platelet-poor plasma (PPP) samples were determined using ELISA and compared. For repetitive freeze-thaw test, PRP was subjected to single (Fr1), double (Fr2), triple (Fr3), or quadruple (Fr4) freeze-thaw cycles and the concentrations of both growth factors in samples were compared similarly. The PDGF-BB concentration in Ca was significantly higher than that in other preparations. The TGF- $\beta 1$ concentrations in $\mathrm{Fr}$ and $\mathrm{Ca}$ were significantly higher than those in PPP and No, with no significant differences between $\mathrm{Fr}$ and $\mathrm{Ca}$. The concentrations of both factors were significantly increased in PRP treated with multiple cycles of freeze-thaw compared with that in PRP treated with a single cycle. No significant differences were noted among $\mathrm{Fr} 2, \mathrm{Fr} 3$, and Fr4. Our findings suggest that activation by adding calcium and autologous serum is optimal for instant use of PRP and that double freeze-thawing is an easier and optimal activation method for cryopreserved PRP.
\end{abstract}

KEY WORDS: equine platelet-rich plasma, freeze-thawing, platelet activation, platelet-derived growth factor-BB (PDGF-BB), transforming growth factor- $\beta 1$ (TGF- $\beta 1$ )

Platelet-rich plasma (PRP) therapy has been widely applied to both human and veterinary medicine $[1,7,15,16,23,33]$ since the innovative report of therapeutic efficacy of PRP by Marx et al [20, 21]. Derived from autologous whole blood, PRP is a concentrated platelet solution in plasma. Platelets in PRP contain various therapeutic ingredients, including platelet-derived growth factor (PDGF), transforming growth factor- $\beta 1$ (TGF- $\beta 1$ ), vascular endothelial growth factor (VEGF), epidermal growth factor (EGF), and fibroblast growth factor (FGF) [7]. Upon activation, these growth factors are released from concentrated platelets and enhance various healing processes around the site of administration [7, 20]. In addition, the use of PRP therapy in humans has been reported for a variety of orthopedic conditions such as osteoarthritis and injuries in tendons, muscles, and ligaments [10, 12, 23]. Currently, it is used to treat similar disorders in veterinary medicine [13, 15, 16, 32, 33].

One of the reasons for the rapid and wide acceptance of PRP therapy is the simplicity of preparing PRP through the double centrifugation method [7, 28, 31,34]. However, Sundman et al. [27] showed that cellular and cytokine compositions in the resulting PRP varied depending on the preparation method and suggested that it influences the biologic effects of PRP. Hence, the cellular composition of PRP that should be prepared according to the conditions is disputable [6, 17, 19, 20, 27].

The concentration of growth factors in PRP depends not only on its composition but also on its activation method [18, 19]. Platelets in PRP are activated by physical or chemical stimuli that cause the release of numerous growth factors, such as those mentioned above $[7,20]$. In equine veterinary practice, two methods are widely used for the activation of PRP: freeze-thawing 
(Fr) to physically disrupt platelet membranes or adding calcium and autologous thrombin-containing serum to induce physiological activation of platelets $(\mathrm{Ca})[3,5]$. Since platelets are activated only by contact with collagen fibers within the extra-cellular matrix in situ [25], it has been thought that activation of PRP is not required before administration. However, Textor et al. showed that administration of resting (nonactivated) PRP results in the release of only a fraction of the growth factors contained within the platelets [31]. In clinical use, a high dose of growth factors is frequently required to immediately enhance the healing process [13]. However, few studies have reported an optimal method of platelet activation to stably and reproducibly maximize growth factor concentrations in PRP, and it is considered essential to standardize the activation method to obtain such PRP preclinically [29].

In this study, we investigated the concentrations of PDGF isoform BB (PDGF-BB) and TGF- $\beta 1$ in equine PRP, which are representative growth factors contained in platelets. In brief, PRP was activated by each of the above mentioned methods or by the repetitive freeze-thawing method. Thereafter, their concentrations in the resulting PRP were determined using ELISA and compared.

\section{MATERIALS AND METHODS}

\section{Animals}

Eleven healthy Thoroughbreds (seven male and four female, $5.0 \pm 1.5$ years old, $496 \pm 26 \mathrm{~kg}$, mean $\pm \mathrm{SD}$ ) were used in this study. All experimental procedures were approved by the Animal Welfare and Ethics Committee of the Equine Research Institute of the Japan Racing Association (authorization numbers 16-7 and 17-8).

\section{Preparation of PRP}

Autologous PRP was prepared using a double-spin method as previously described [8]. One hundred $\mathrm{m} l$ of equine whole blood was collected from the jugular vein of each of the 11 Thoroughbred horses using a disposable plastic syringe containing $10 \%$ sodium citrate anticoagulant (ACD-A injection, Terumo BCT Ltd., Tokyo, Japan). Equal amounts of blood were dispensed into ten polypropylene tubes and centrifuged at $400 \times g$ for $7 \mathrm{~min}$ at $4^{\circ} \mathrm{C}$. The plasma fraction of each tube was transferred into another polypropylene tube and centrifuged at $2,000 \times g$ for $7 \mathrm{~min}$ at $4^{\circ} \mathrm{C}$. The supernatant was removed and kept as platelet-poor plasma (PPP). One $\mathrm{m} l$ of supernatant was left in the bottom of each tube and used to resuspend the pellet for the preparation of PRP. The concentrations of platelets and leukocytes in the PRP and PPP were determined using an automated blood cell counter (Sysmex K-4500, Sysmex Corp., Kobe, Japan). The PRP samples were randomly divided into two groups and used for an activation test $(n=6)$ or a repetitive freeze-thaw test $(n=5)$. Concurrently, autologous serum was prepared for the activation of PRP by centrifuging peripheral blood in a blood collection tube (BD Vacutainer SST ${ }^{\mathrm{TM}}$ II Advance Tube, BD Limited, Plymouth, UK) at 2,000 $\times g$ for $10 \mathrm{~min}$ at $4^{\circ} \mathrm{C}$.

\section{PRP activation test}

Two different activation methods were conducted. In the $\mathrm{Fr}$ method, the prepared PRP was frozen at $-80^{\circ} \mathrm{C}$ for $2 \mathrm{hr}$ and completely thawed at room temperature for $30 \mathrm{~min}$. In the $\mathrm{Ca}$ method, a mixture of $10 \% \mathrm{CaCl}_{2}$ solution and autologous serum at a ratio of $1: 3$ was mixed in a 1:10 ratio with PRP in a microtube. The mixed PRP was allowed to gel by incubation at $37^{\circ} \mathrm{C}$ for $5 \mathrm{~min}$. The gelled PRP was centrifuged at $10,000 \times g$ for $5 \mathrm{~min}$ at $4^{\circ} \mathrm{C}$ before collection of the supernatant. Similarly, $\mathrm{Fr}$ and nonactivated $(\mathrm{No}$ ) samples were centrifuged under the same conditions before collection of the supernatant. $\mathrm{Ca}, \mathrm{Fr}, \mathrm{No}$, and PPP supernatants were preserved at $4^{\circ} \mathrm{C}$ for the analysis of growth factors.

The concentrations of PDGF-BB and TGF- $\beta 1$ in samples were determined in duplicate using ELISA kits (Quantikine Human PDGF-BB ELISA DBB00 and Quantikine Human TGF- $\beta 1$ ELISA DB100B, R\&D Systems, Minneapolis, MN, USA) according to the manufacturer's instructions. These kits were designed for testing human samples but have also been validated for use in horses $[3,11,28,30,31]$. All procedures were performed within $6 \mathrm{hr}$ of preparation to ensure the stability of growth factors [11].

To observe morphological changes in platelets and leukocytes, smears of PPP, $\mathrm{No}, \mathrm{Fr}$, and $\mathrm{Ca}$ supernatants were stained using a commercial kit (Diff-Quick staining kit, Sysmex Corp.) and examined microscopically.

\section{PRP repetitive freeze-thaw test}

PRP samples were frozen for 1 month and were subjected to single $(F r 1)$, double $(F r 2)$, triple $(F r 3)$, or quadruple $(F r 4)$ freezethaw cycles, with repeated overnight freezing at $-30^{\circ} \mathrm{C}$ and thawing at room temperature. All samples were divided into two aliquots after each freeze-thaw cycle (Fig. 1). One aliquot was centrifuged at $10,000 \times g$ for 5 min at $4^{\circ} \mathrm{C}$ before collection of the supernatant. The remaining precipitate was dissolved and incubated for $30 \mathrm{~min}$ at room temperature with $0.5 \%$ Triton ${ }^{\mathrm{TM}} \mathrm{X}-100$ (SIGMA-ALDRICH, Co., St. Louis, MO, USA) PBS solution containing a protease inhibitor (cOmplete ${ }^{\mathrm{TM}}$ ULTRA Tablets, Roche Ltd., Basel, Switzerland). To dissolve platelet membranes and determine the total growth factor content in PRP samples, another aliquot was incubated for $30 \mathrm{~min}$ at room temperature using the Triton buffer described above (washed $\mathrm{Fr}$ ). The concentrations of PDGF-BB and TGF- $\beta 1$ in the supernatant, dissolved precipitate, and washed $F r$ (total concentration) were determined in the same manner as described previously and compared.

One half of the supernatant was frozen at $-30^{\circ} \mathrm{C}$ overnight and thawed at room temperature for 30 min (treated supernatant) to determine the effect of the freeze-thaw treatment itself on growth factor concentration. The concentrations of PDGF-BB and TGF- $\beta 1$ in these samples were also determined and compared to the concentrations in the pre-treated supernatant samples. Additionally, to clarify the amount of growth factors accumulated during freeze-thawing, the concentrations of growth factors 


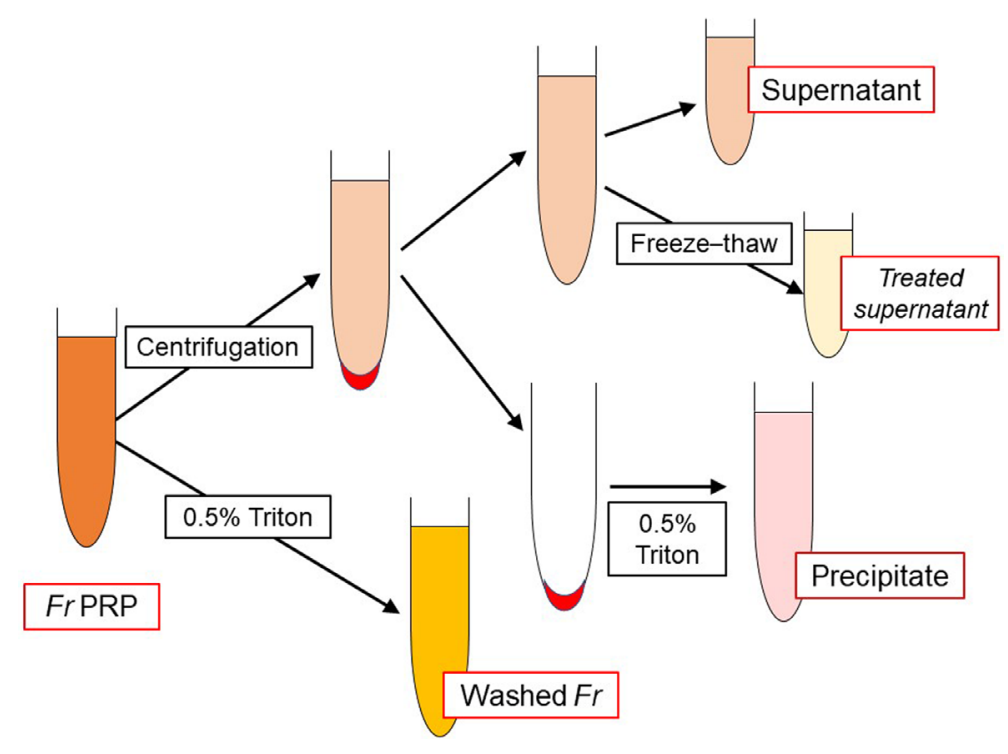

Fig. 1. Schematic representation of the platelet-rich plasma (PRP) repetitive freeze-thaw test. Fr PRP: freeze-thawing PRP.

in supernatants collected after freeze-thawing PRP were compared with those in freeze-thawing PRP supernatant (treated supernatant). Smear examination was also performed for each $F r$ sample as mentioned above.

\section{Statistical analysis}

Growth factor concentrations were compared using one-way ANOVA, and post-hoc analyses were performed using Tukey's test. Concentration ratios were compared using the Kruskal-Wallis test, and the subsequent post-hoc analysis was performed using the Steel-Dwass test. Concentrations of the freeze-thawing supernatants were compared using paired $t$-tests. Analyses were performed using Microsoft Excel 2013 Macro applications (Excel TOKEI ver. 7.0, ESUMI Co., Ltd., Tokyo, Japan). Statistical significance was set at $P<0.05$.

\section{RESULTS}

Platelet and leukocyte counts in whole blood and PRP are shown in Table 1. Microscopic examination of smear samples revealed that leukocytes in PRP predominantly comprised lymphocytes (approximately $>90 \%$ ). Neither leukocytes nor platelets were observed in PPP or the supernatants of $C a$ gel. Platelets appeared to maintain their morphology, whereas leukocytes were not observed in Fr1, Fr2, and Fr3 (Fig. 2).

\section{PRP activation test}

The concentration of PDGF-BB in PPP and No was below the linear range of measurement $(<31.2 \mathrm{pg} / \mathrm{m} l)$. The concentration of PDGF-BB in $C a(5,222 \pm 3,957 \mathrm{pg} / \mathrm{ml}$, mean $\pm \mathrm{SD})$ was significantly higher than that in other preparations $(F r, 1,369 \pm 1,138 \mathrm{pg} /$ $\mathrm{ml}$, Fig. 3A). The concentrations of TGF- $\beta 1$ in $F r(7,235 \pm 2,842 \mathrm{pg} / \mathrm{m} l)$ and $C a(8,084 \pm 2,257 \mathrm{pg} / \mathrm{m} l)$ were significantly higher than those in PPP $(1,779 \pm 461 \mathrm{pg} / \mathrm{ml})$ and $N o(1,994 \pm 442 \mathrm{pg} / \mathrm{m} l)$, with no significant differences between $\mathrm{Fr}$ and $\mathrm{Ca}$ (Fig. 3B).

\section{$P R P$ repetitive freeze-thaw test}

The total PDGF-BB concentrations in Fr, 1 Fr2, Fr3, and Fr4 samples $(9,486 \pm 553 \mathrm{pg} / \mathrm{ml}, 11,545 \pm 1,873 \mathrm{pg} / \mathrm{ml}, 12,295 \pm$ $1,709 \mathrm{pg} / \mathrm{ml}$, and $11,288 \pm 1,591 \mathrm{pg} / \mathrm{ml}$, respectively) were significantly higher than those in the respective supernatants $(2,698 \pm$ $1,510 \mathrm{pg} / \mathrm{ml}, 5,224 \pm 913 \mathrm{pg} / \mathrm{ml}, 6,294 \pm 1,254 \mathrm{pg} / \mathrm{ml}$, and 6,351 $\pm 1,419 \mathrm{pg} / \mathrm{ml}$, respectively) and precipitates $(3,512 \pm 2,045 \mathrm{pg} /$ $\mathrm{ml}, 2,906 \pm 654 \mathrm{pg} / \mathrm{ml}, 2,236 \pm 344 \mathrm{pg} / \mathrm{ml}$, and 2,069 $\pm 572 \mathrm{pg} / \mathrm{ml}$, respectively) (Fig. 4A). No significant difference was observed between the Frl supernatant and precipitate. However, in the Fr2, Fr3, and Fr4 samples, the concentration of PDGF-BB in supernatants was significantly higher than that in precipitates. The concentrations of PDGF-BB in the Frl and Fr2 supernatants were significantly decreased by freeze-thawing with concentrations in the Fr1 and Fr2 treated supernatants of 2,154 $\pm 1,188$ pg/ $\mathrm{m} l$ and $4,218 \pm 852 \mathrm{pg} / \mathrm{ml}$, respectively, which were significantly lower than those in freeze-thawing PRP supernatant (Fig. 4B). The concentrations of PDGF-BB in the Fr2, Fr3, and Fr4 supernatants were significantly higher than that in the Fr1 supernatant, whereas no significant differences in PDGF-BB concentration were noted among the Fr2, Fr3, and Fr4 supernatants (Fig. 4C). There were no significant differences in precipitate or total PDGF-BB concentrations among the four groups.

There were no significant differences in TGF- $\beta 1$ concentrations within samples of the same freeze-thaw cycle number, except in total concentration of the Fr4 $(55,506 \pm 22,461 \mathrm{pg} / \mathrm{ml})$, which was significantly higher than that of the precipitate of the $\mathrm{Fr} 4$ $(15,166 \pm 3,210 \mathrm{pg} / \mathrm{ml})$ (Fig. 4D). The concentration of TGF- $\beta 1$ in the $F r 3$ supernatant $(40,386 \pm 14,338 \mathrm{pg} / \mathrm{ml})$ was significantly 
Table 1. The summary of obtained platelet-rich plasma (PRP) $(n=11)$

\begin{tabular}{lcc}
\hline & $\begin{array}{c}\text { Leukocyte } \\
\left(\times 10^{6} / \mathrm{m} l\right)\end{array}$ & $\begin{array}{c}\text { Platelet } \\
\left(\times 10^{8} / \mathrm{m} l\right)\end{array}$ \\
\hline Whole blood & $6.7 \pm 1.2$ & $1.2 \pm 0.2$ \\
PRP & $3.7 \pm 1.6$ & $9.2 \pm 1.6$ \\
Concentration ratio & $0.6 \pm 0.3$ & $7.4 \pm 0.9$ \\
\hline
\end{tabular}

Mean $\pm \mathrm{SD}$. Concentration ratio is the value of $\mathrm{PRP} /$ whole blood.
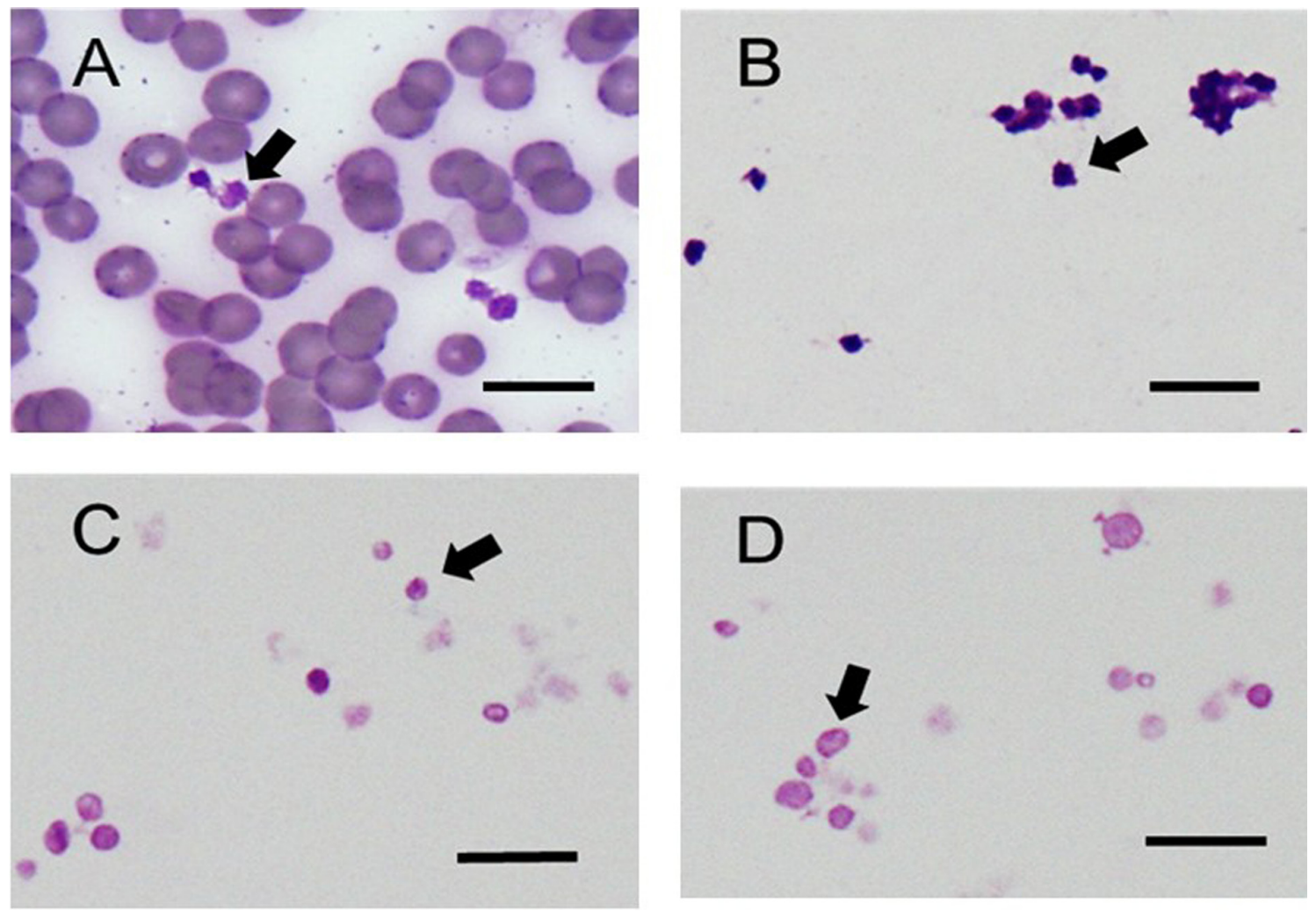

Fig. 2. Micrographs of Diff-Quick-stained whole blood smears (A), fresh platelet-rich plasma (PRP) (B), single freeze-thawing PRP (C), and triple freeze-thawing PRP (D). Platelets maintaining native morphology are indicated with black arrows. Bars=10 $\mu \mathrm{m}$.
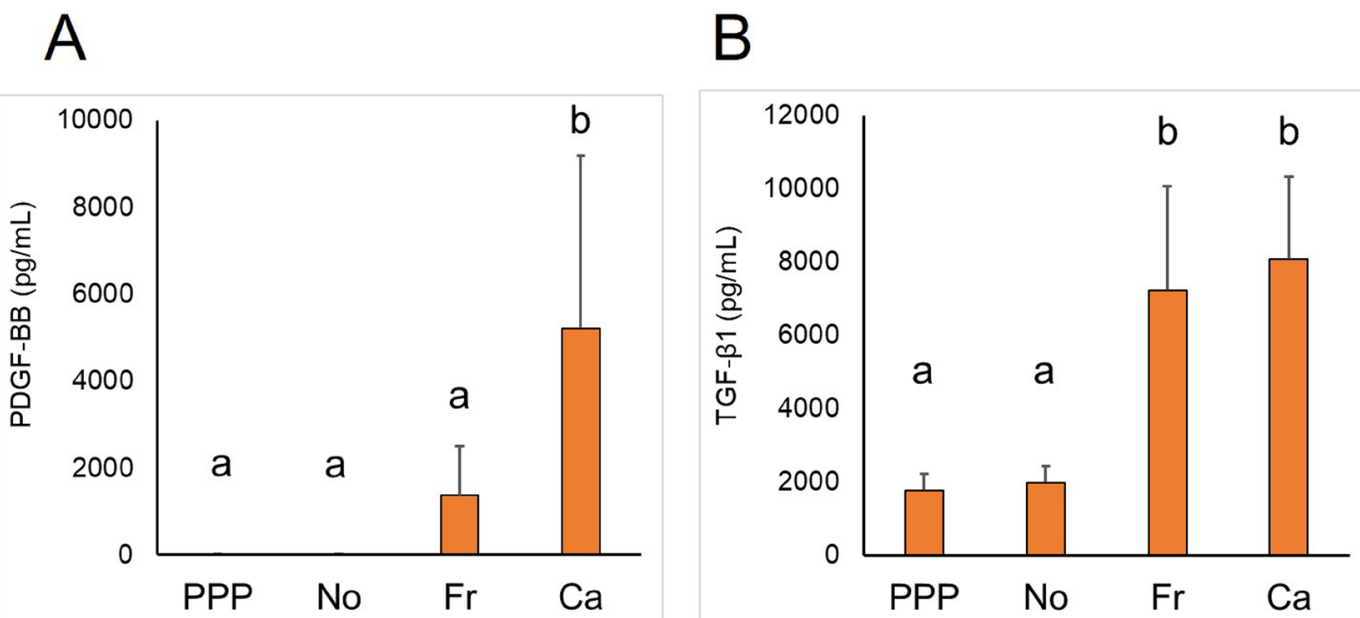

Fig. 3. The concentrations of platelet-derived growth factor-BB (PDGF-BB) (A) and transforming growth factor- $\beta 1$ (TGF- $\beta 1)(B)$ in platelet-poor plasma (PPP), nonactivated platelet-rich plasma (PRP) (No), freeze-thawing PRP ( Fr), and PRP to which calcium and autologous serum were added $(\mathrm{Ca})$ (mean $\pm \mathrm{SD}, \mathrm{n}=6)$. Different letters indicate significant differences among groups $(P<0.05)$. 
A
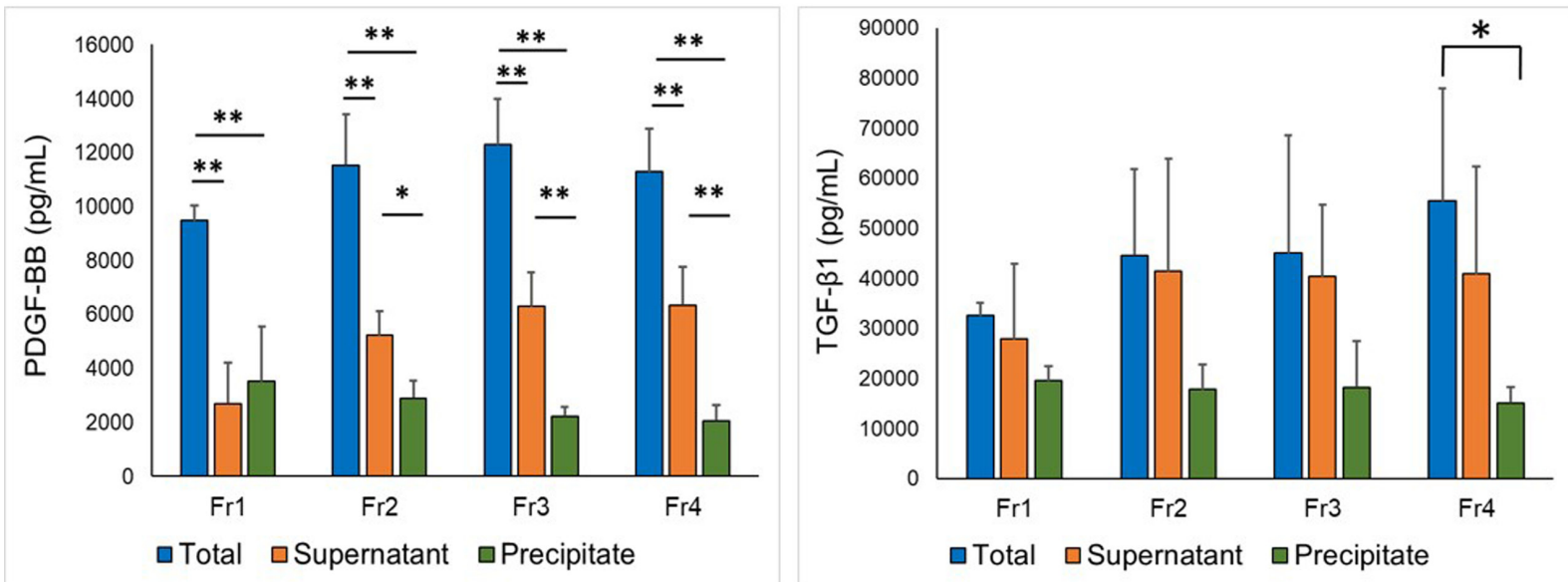

B

E

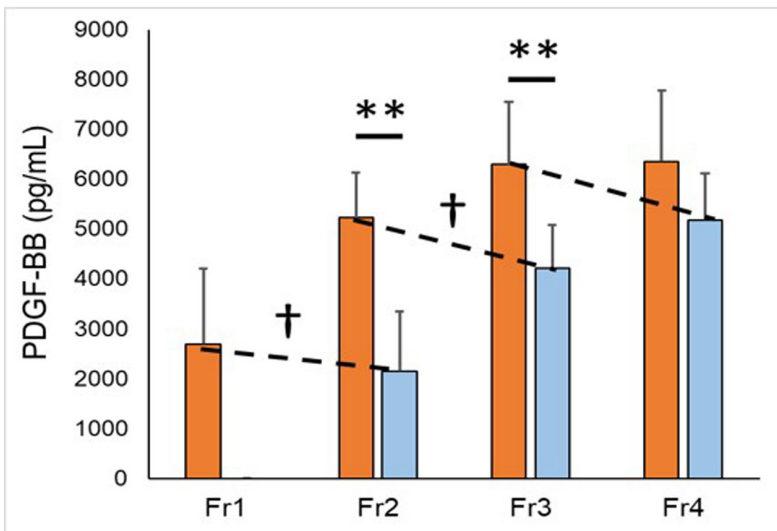

$\square$ Freeze-thawing PRP $\square$ Treated supernatant

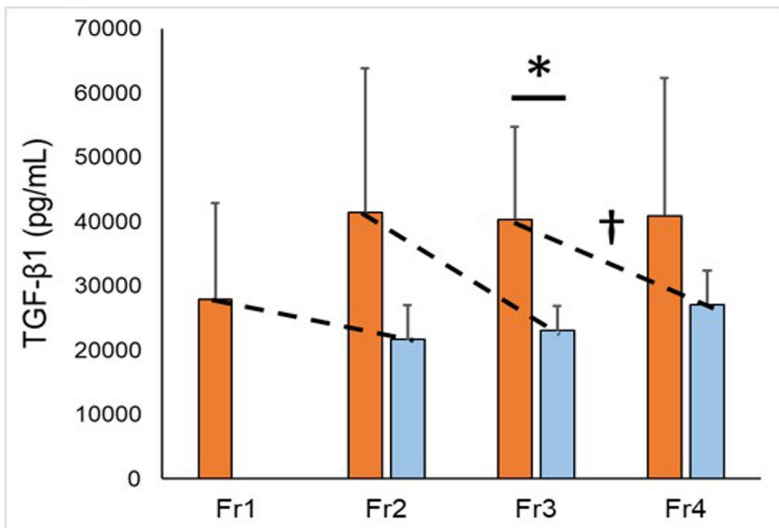

$\square$ Freeze-thawing PRP $\square$ Treated supernatant

C

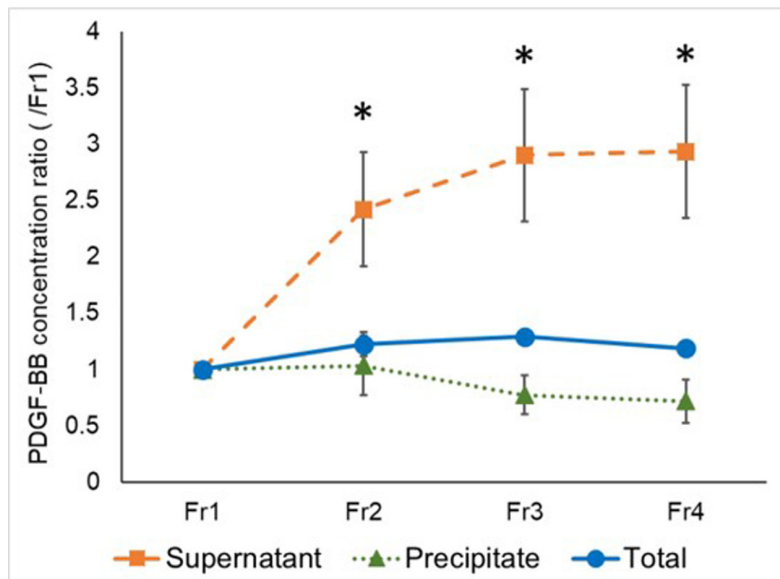

$\mathrm{F}$

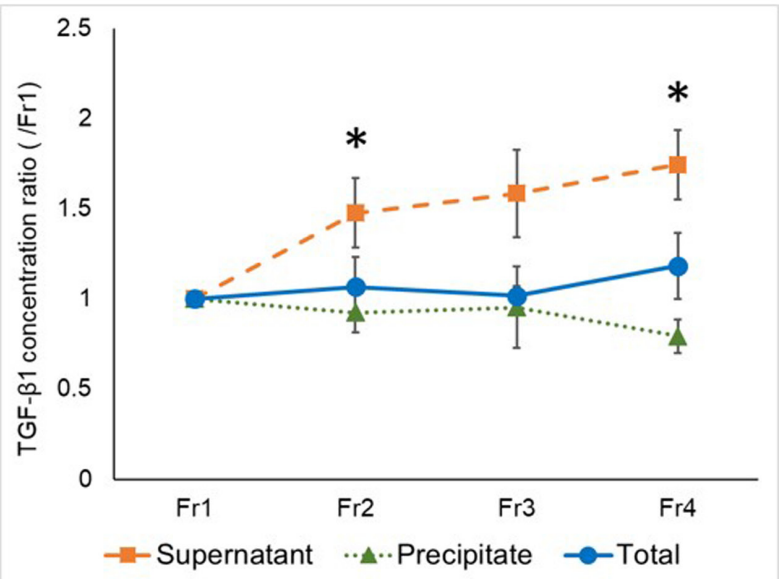

Fig. 4. Platelet-derived growth factor-BB (PDGF-BB) (A) and transforming growth factor- $\beta 1$ (TGF- $\beta 1)$ (D) concentrations in single (Fr1), double $(F r 2)$, triple ( $F r 3$ ), and quadruple ( Fr 4 ) freeze-thawing platelet-rich plasma (PRP) (mean $\pm \mathrm{SD}, \mathrm{n}=5$ ). Comparisons among total concentration, concentration in the supernatant, and concentration in the precipitate obtained after centrifugation $(* P<0.05, * * P<0.01)$. PDGF-BB (B) and TGF- $\beta 1$ (E) concentration in freeze-thawing PRP supernatant (mean \pm SD). Freeze-thawing PRP in (B) and (E)=supernatant in (A) and (D), respectively. Dashed lines indicate comparisons of the supernatant concentrations before and after freeze-thawing $(*, \dagger P<0.05 ; * *, \dagger \dagger P<0.01)$. PDGF-BB (C) or TGF- $\beta 1$ (F) concentration ratios compared to Frl (mean \pm SE). (C) The concentrations in the Fr2, Fr3, and Fr4 supernatants were significantly higher than that in the $F r l$ supernatant. (F) The concentrations in the $F r 2$ and $F r 4$ supernatants were significantly higher than that in the Fr1 supernatant. No significant differences were observed among the Fr2, Fr3, and $F r 4$ supernatants $\left({ }^{*} P<0.05\right)$. 
decreased by freeze-thawing with concentrations in the $F r 3$ treated supernatants of 27,074 $\pm 5,336 \mathrm{pg} / \mathrm{ml}$ (Fig. 4E). The Fr3 supernatant contained significantly higher levels of TGF- $\beta 1$ than the freeze-thawing Fr 2 supernatant $(23,044 \pm 3,887 \mathrm{pg} / \mathrm{ml})$. The concentrations of TGF- $\beta 1$ in the $F r 2$ and $F r 4$ supernatants were significantly higher than that in the $F r 1$ supernatant, whereas no significant differences in TGF- $\beta 1$ concentration were noted among the Fr2, Fr3, and Fr4 supernatants (Fig. 4F). There were no significant differences in precipitate or total TGF- $\beta 1$ concentrations among the four groups.

\section{DISCUSSION}

There are many methods for preparing PRP with various cellular and molecular components. Furthermore, the methods of PRP activation are diverse [29]. Consequently, although PRP therapy is widely applied in human and equine orthopedic surgery, the clinical outcomes vary $[4,24,29]$. Because instantly effective ingredients are present in the supernatant of activated PRP, we examined an activation method to stably maximize the growth factor concentration in PRP supernatant. In addition, we posited that the variation in outcomes can be minimized by using such PRP for treatment.

Upon injury, PDGF stimulates mitogenicity and chemotaxis of leukocytes, fibroblasts, and smooth muscle cells to the wound site, and also enhances angiogenesis via upregulation of $V E G F$ gene expression [2]. TGF- $\beta 1$ also plays a crucial role in angiogenesis and connective tissue regeneration in wound healing. Additionally, TGF- $\beta 1$ is a potent inhibitor of metalloproteinase preventing collagen breakdown [2]. In humans, because the levels of these growth factors are decreased in chronic wounds, recombinant human variants of PDGF-BB have been successfully administered for such lesions [2]. In equine practice, because autologous growth factor therapy may be considered a safer application than xenogeneic therapy [30], it seems clinically important to use PRP with high concentrations of these growth factors for treatment.

PRP activation tests revealed that activated PRP contains a high concentration of growth factors regardless of the activation method. Activation of PRP by the $C a$ method resulted in the highest PDGF-BB concentrations. In contrast, no significant differences in TGF- $\beta 1$ concentrations between the $F r$ and $C a$ methods were observed. Similar conclusions have been reported by Textor et al [30].

Our results suggest that single freeze-thawing PRP contains an abundance of PDGF-BB in the precipitate, similar to that in the supernatant. Therefore, it is likely that the single freeze-thaw method is insufficient to entirely release PDGF-BB from platelets in PRP. Compared with the addition of calcium and serum, this method results in lower levels of PDGF-BB. However, there was no difference in the TGF- $\beta 1$ concentration between the $F r$ and $C a$ methods. There was a large variation in the TGF- $\beta 1$ concentrations between individual PRP supernatants activated by the single freeze-thaw method. It was previously reported that TGF- $\beta 1$ from disrupted leukocytes contributes to its concentration in PRP [35]. In this study, although we attempted to prepare PRP in such a manner that the presence of leukocytes was kept as low as possible, the contamination of low-dose leukocytes was inevitable. We considered the possibility that the variation in TGF- $\beta 1$ concentration among individual samples was due to a non-negligible amount of TGF- $\beta 1$ released from leukocytes, as has been previously reported in humans [35]. Consequently, we did not observe differences in TGF- $\beta 1$ concentrations corresponding to the activation method. Our results show that a method involving the addition of calcium and autologous serum may be superior for activation of PRP for instant use. However, the concentrations of growth factors in $\mathrm{Ca}$ supernatants showed high variability. Therefore, it was considered important to establish an activation method capable of more stably attaining high growth factor concentrations.

Our results also suggested that repeated freeze-thaw cycles result in higher growth factor concentrations in the supernatant of PRP than that obtained by a single freeze-thaw cycle. In humans, it was reported that double freeze-thawing results in higher PDGF concentrations in PRP supernatant than that obtained by single freeze-thawing [35]. It was also reported that the concentrations of PDGF-BB, TGF- $\beta 1$, EGF, and FGF increased by repetitive freeze-thawing and plateaued at 3 and 5 cycles [26]. We found that the supernatants of PRP that were freeze-thawed multiple times contained significantly higher concentrations of growth factors than those subjected to single freeze-thawing. On the other hand, no significant differences were noted among PRP that was freeze-thawed two or more times. We hypothesized that concentrations of both PDGF-BB and TGF- $\beta 1$ would increase in PRP supernatants because the number of freeze-thaw cycles should cause increased disruption of platelets and growth factor release. However, we found that the concentration of neither growth factor was increased with increasing cycles of freeze-thaw. We speculate that PDGF-BB is considerably vulnerable to degradation upon freeze-thawing. Conversely, the increase in growth factor concentrations in freeze-thawing PRP is caused by de-novo release of growth factors from disrupted platelets remaining in the precipitate. Therefore, it is reasonable to hypothesize that additional cycles do not significantly affect growth factor concentration because amounts lost and produced are in equilibrium. Conversely, TGF- $\beta 1$ is highly tolerant to degradation upon freeze-thawing, as has been reported in humans $[9,14]$. Therefore, freeze-thawing of PRP resulted in the accumulation of newly released TGF- $\beta 1$. In contrast, even after repeated freeze-thawing, there was no clear difference in TGF- $\beta 1$ concentrations among the total, supernatant, and precipitate samples. It is reasonable to assume that the TGF- $\beta 1$ concentration in PRP depends not only on platelets but also on other components, such as leukocytes [35]. Hence, no significant differences were observed among the Fr2, Fr3, and Fr4 supernatants. Based on our findings, the optimal growth factor concentration in PRP supernatant may be obtained by freezing and thawing at least twice.

Growth factor concentrations in Frl samples were higher than those in Fr samples in the activation test, although these were similar supernatants activated by the single freeze-thaw method. McClain and McCarrel indicated that the growth factor content in equine PRP varies with long-term cryopreservation [22]. In our activation test, it was necessary to determine the concentrations of growth factors in PRP preparations within $6 \mathrm{hr}$ in order to measure stable concentrations consistently between all sample types 
[11]. In contrast, frozen PRP stored for at least 1 month was thawed and measured in the repetitive freeze-thaw test, which may be the cause of the difference in values obtained. We found that high concentrations of growth factors can be maintained even after 1 month of PRP cryopreservation. Furthermore, 2.4-fold higher PDGF-BB and 1.5-fold higher TGF- $\beta 1$ concentrations were obtained using double freeze-thawing for activation than by using single freeze-thawing. This indicates that double freeze-thawing can result in growth factor concentrations and stability that are comparable to calcium and serum addition. Consequently, PRP activated by the $\mathrm{Ca}$ method can be applied quickly and appears to be suitable for instant use. However, growth factors once extracted into the supernatant are prone to inactivation. Hence, if activated PRP is intended for repeated long-term use, it is easier to prepare PRP in large cryopreserved batches by dispensing it in small quantities and double freeze-thawing when used.

In this study, we investigated only two growth factors and did not investigate other components in PRP. On the other hand, as mentioned above, it is known that these two growth factors, which are abundant in platelets, are essential contributors to the wound healing process. Therefore, when considering the clinical effects of PRP therapy, these growth factors must be focused on. However, further studies are needed to determine the appropriate application method of equine PRP optimally prepared and activated for clinical use [29]. In conclusion, our findings suggest that activation by adding calcium and autologous serum is optimal for instant use of PRP and that double freeze-thawing is an easier and optimal activation method for cryopreserved PRP.

\section{REFERENCES}

1. Alio, J. L., Rodriguez, A. E., Abdelghany, A. A. and Oliveira, R. F. 2017. Autologous platelet-rich plasma eye drops for the treatment of postLASIK chronic ocular surface syndrome. J. Ophthalmol. 2017: 2457620. [Medline] [CrossRef]

2. Barrientos, S., Stojadinovic, O., Golinko, M. S., Brem, H. and Tomic-Canic, M. 2008. Growth factors and cytokines in wound healing. Wound Repair Regen. 16: 585-601. [Medline] [CrossRef]

3. Bosch, G., van Schie, H. T., de Groot, M. W., Cadby, J. A., van de Lest, C. H., Barneveld, A. and van Weeren, P. R. 2010. Effects of platelet-rich plasma on the quality of repair of mechanically induced core lesions in equine superficial digital flexor tendons: A placebo-controlled experimental study. J. Orthop. Res. 28: 211-217. [Medline]

4. Brossi, P. M., Moreira, J. J., Machado, T. S. and Baccarin, R. Y. 2015. Platelet-rich plasma in orthopedic therapy: a comparative systematic review of clinical and experimental data in equine and human musculoskeletal lesions. BMC Vet. Res. 11: 98. [Medline] [CrossRef]

5. DeRossi, R., Coelho, A. C., Mello, G. S., Frazílio, F. O., Leal, C. R., Facco, G. G. and Brum, K. B. 2009. Effects of platelet-rich plasma gel on skin healing in surgical wound in horses. Acta Cir. Bras. 24: 276-281. [Medline] [CrossRef]

6. Dhurat, R. and Sukesh, M. 2014. Principles and methods of preparation of platelet-rich plasma: A review and author's perspective. J. Cutan. Aesthet. Surg. 7: 189-197. [Medline] [CrossRef]

7. Everts, P. A., Knape, J. T., Weibrich, G., Schönberger, J. P., Hoffmann, J., Overdevest, E. P., Box, H. A. and van Zundert, A. 2006. Platelet-rich plasma and platelet gel: a review. J. Extra Corpor. Technol. 38: 174-187. [Medline]

8. Fukuda, K., Miyata, H., Kuwano, A., Kuroda, T., Tamura, N., Kotoyori, Y. and Kasashima, Y. 2017. Does the injection of platelet-rich plasma induce changes in the gene expression and morphology of intact Thoroughbred skeletal muscle? J. Equine Sci. 28: 31-39. [Medline] [CrossRef]

9. Giles, B. M., Underwood, T. T., Benhadji, K. A., Nelson, D. K., Grobeck, L. M., Lin, B., Wang, S., Fill, J. A., Man, M., Pitts, K. R. and Bamberg, A. 2018. Analytical characterization of an enzyme-linked immunosorbent assay for the measurement of transforming growth factor $\beta 1$ in human plasma. J. Appl. Lab. Med. 3: 200-212. [CrossRef]

10. Hammond, J. W., Hinton, R. Y., Curl, L. A., Muriel, J. M. and Lovering, R. M. 2009. Use of autologous platelet-rich plasma to treat muscle strain injuries. Am. J. Sports Med. 37: 1135-1142. [Medline] [CrossRef]

11. Hauschild, G., Geburek, F., Gosheger, G., Eveslage, M., Serrano, D., Streitbürger, A., Johannlükens, S., Menzel, D. and Mischke, R. 2017. Short term storage stability at room temperature of two different platelet-rich plasma preparations from equine donors and potential impact on growth factor concentrations. BMC Vet. Res. 13: 7. [Medline] [CrossRef]

12. Hsu, W. K., Mishra, A., Rodeo, S. R., Fu, F., Terry, M. A., Randelli, P., Canale, S. T. and Kelly, F. B. 2013. Platelet-rich plasma in orthopaedic applications: evidence-based recommendations for treatment. J. Am. Acad. Orthop. Surg. 21: 739-748. [Medline] [CrossRef]

13. Iacopetti, I., Perazzi, A., Ferrari, V. and Busetto, R. 2012. Application of platelet-rich gel to enhance wound healing in the horse: A case report. $J$. Equine Vet. Sci. 32: 123-128. [CrossRef]

14. Ito, Y., Nakachi, K., Imai, K., Hashimoto, S., Watanabe, Y., Inaba, Y., Tamakoshi, A., Yoshimura T., JACC Study Group 2005. Stability of frozen serum levels of insulin-like growth factor-I, insulin-like growth factor-II, insulin-like growth factor binding protein-3, transforming growth factor beta, soluble Fas, and superoxide dismutase activity for the JACC study. J. Epidemiol. 15 Suppl 1: S67-S73. [Medline] [CrossRef]

15. Jee, C. H., Eom, N. Y., Jang, H. M., Jung, H. W., Choi, E. S., Won, J. H., Hong, I. H., Kang, B. T., Jeong, D. W. and Jung, D. I. 2016. Effect of autologous platelet-rich plasma application on cutaneous wound healing in dogs. J. Vet. Sci. 17: 79-87. [Medline] [CrossRef]

16. Kim, J. H., Park, C. and Park, H. M. 2009. Curative effect of autologous platelet-rich plasma on a large cutaneous lesion in a dog. Vet. Dermatol. 20: 123-126. [Medline] [CrossRef]

17. Kushida, S., Kakudo, N., Morimoto, N., Hara, T., Ogawa, T., Mitsui, T. and Kusumoto, K. 2014. Platelet and growth factor concentrations in activated platelet-rich plasma: a comparison of seven commercial separation systems. J. Artif. Organs 17: 186-192. [Medline] [CrossRef]

18. Lana, J. F. S. D., Purita, J., Paulus, C., Huber, S. C., Rodrigues, B. L., Rodrigues, A. A., Santana, M. H., Madureira, J. L. Jr., Malheiros Luzo, Â. C., Belangero, W. D. and Annichino-Bizzacchi, J. M. 2017. Contributions for classification of platelet rich plasma - proposal of a new classification: MARSPILL. Regen. Med. 12: 565-574. [Medline] [CrossRef]

19. Magalon, J., Chateau, A. L., Bertrand, B., Louis, M. L., Silvestre, A., Giraudo, L., Veran, J. and Sabatier, F. 2016. DEPA classification: a proposal for standardising PRP use and a retrospective application of available devices. BMJ Open Sport Exerc. Med. 2: e000060. [Medline] [CrossRef]

20. Marx, R. E. 2004. Platelet-rich plasma: evidence to support its use. J. Oral Maxillofac. Surg. 62: 489-496. [Medline] [CrossRef]

21. Marx, R. E., Carlson, E. R., Eichstaedt, R. M., Schimmele, S. R., Strauss, J. E. and Georgeff, K. R. 1998. Platelet-rich plasma: Growth factor enhancement for bone grafts. Oral Surg. Oral Med. Oral Pathol. Oral Radiol. Endod. 85: 638-646. [Medline] [CrossRef]

22. McClain, A. K. and McCarrel, T. M. 2019. The effect of four different freezing conditions and time in frozen storage on the concentration of commonly measured growth factors and enzymes in equine platelet-rich plasma over six months. BMC Vet. Res. 15: 292. [Medline] [CrossRef]

23. Mei-Dan, O., Lippi, G., Sánchez, M., Andia, I. and Maffulli, N. 2010. Autologous platelet-rich plasma: a revolution in soft tissue sports injury 
management? Phys. Sportsmed. 38: 127-135. [Medline] [CrossRef]

24. Navani, A., Li, G. and Chrystal, J. 2017. Platelet rich plasma in musculoskeletal pathology: A necessary rescue or a lost cause? Pain Physician 20: E345-E356. [Medline] [CrossRef]

25. Sano, K., Takai, Y., Yamanishi, J. and Nishizuka, Y. 1983. A role of calcium-activated phospholipid-dependent protein kinase in human platelet activation. Comparison of thrombin and collagen actions. J. Biol. Chem. 258: 2010-2013. [Medline]

26. Strandberg, G., Sellberg, F., Sommar, P., Ronaghi, M., Lubenow, N., Knutson, F. and Berglund, D. 2017. Standardizing the freeze-thaw preparation of growth factors from platelet lysate. Transfusion 57: 1058-1065. [Medline] [CrossRef]

27. Sundman, E. A., Cole, B. J. and Fortier, L. A. 2011. Growth factor and catabolic cytokine concentrations are influenced by the cellular composition of platelet-rich plasma. Am. J. Sports Med. 39: 2135-2140. [Medline] [CrossRef]

28. Sutter, W. W., Kaneps, A. J. and Bertone, A. L. 2004. Comparison of hematologic values and transforming growth factor- $\beta$ and insulin-like growth factor concentrations in platelet concentrates obtained by use of buffy coat and apheresis methods from equine blood. Am. J. Vet. Res. 65: 924-930. [Medline] [CrossRef]

29. Tambella, A. M., Martin, S., Cantalamessa, A., Serri, E. and Attili, A. R. 2018. Platelet-rich plasma and other hemocomponents in veterinary regenerative medicine. Wounds 30: 329-336. [Medline]

30. Textor, J. A. and Tablin, F. 2012. Activation of equine platelet-rich plasma: comparison of methods and characterization of equine autologous thrombin. Vet. Surg. 41: 784-794. [Medline] [CrossRef]

31. Textor, J. A., Norris, J. W. and Tablin, F. 2011. Effects of preparation method, shear force, and exposure to collagen on release of growth factors from equine platelet-rich plasma. Am. J. Vet. Res. 72: 271-278. [Medline] [CrossRef]

32. Waselau, M., Sutter, W. W., Genovese, R. L. and Bertone, A. L. 2008. Intralesional injection of platelet-rich plasma followed by controlled exercise for treatment of midbody suspensory ligament desmitis in Standardbred racehorses. J. Am. Vet. Med. Assoc. 232: 1515-1520. [Medline] [CrossRef]

33. Xie, X., Zhao, S., Wu, H., Xie, G., Huangfu, X., He, Y. and Zhao, J. 2013. Platelet-rich plasma enhances autograft revascularization and reinnervation in a dog model of anterior cruciate ligament reconstruction. J. Surg. Res. 183: 214-222. [Medline] [CrossRef]

34. Yin, W., Xu, H., Sheng, J., Zhu, Z., Jin, D., Hsu, P., Xie, X. and Zhang, C. 2017. Optimization of pure platelet-rich plasma preparation: A comparative study of pure platelet-rich plasma obtained using different centrifugal conditions in a single-donor model. Exp. Ther. Med. 14 2060-2070. [Medline] [CrossRef]

35. Zimmermann, R., Arnold, D., Strasser, E., Ringwald, J., Schlegel, A., Wiltfang, J. and Eckstein, R. 2003. Sample preparation technique and white cell content influence the detectable levels of growth factors in platelet concentrates. Vox Sang. 85: 283-289. [Medline] [CrossRef] 